\title{
A saúde das crianças dos Estados do Ceará, Rio Grande do Norte e Sergi- pe, Brasil: descrição de uma metodologia para diagnósticos comunitários*
}

\author{
Child health in the States of Ceará, Rio Grande do Norte and Sergipe, Brazil: \\ description of a methodology for the diagnosis of communities
}

\begin{abstract}
Cesar G. Victora ${ }^{* *},{ }^{* *}$, Fernando C. Barros** ${ }^{* * *}$, Elaine Tomasi ${ }^{* *}$, Francisca Selma Ferreira****, Jay MacAuliffe $e^{* * *}$, Anamaria Cavalcante Silva******, Francisca Maria Andrade*****, Lidia Wi-

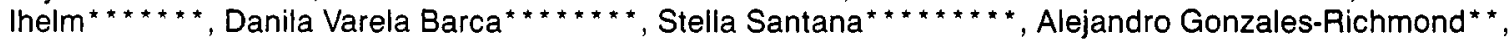
Roger Shrimpton**
\end{abstract}

VICTORA, C.G. et al. A saúde das crianças dos Estados do Ceará, Rio Grande do Norte e
Sergipe, Brasil: descrição de uma metodologia para diagnósticos comunitários. Rev. Saúde
públ., S. Paulo, 25: $218-25,1991$. Para a maior parte dos estados brasileiros, inexistem indica-
dores confiáveis sobre a saúde das crianças - tais como estado nutricional, aleitamento, cober-
tura vacinal, frequência e manejo de doenças infecciosas e cobertura de serviço de atenção
pré e perinatal. Para obter tais informações, desenvolveu-se uma metodologia para diagnosti-
cos a nivel estadual, aplicada recentemente em amostras representativas nos Estados do Cea-
rá, Sergipe e Rio Grande do Norte. O presente artigo descreve os principais aspectos desta
metodologia e alguns de seus achados mais relevantes. Os resultados obtidos apontam para
a necessidade de incentivar o aleitamento materno, aumentar a cobertura vacinal, incremen-
tar o uso da terapia de reidratação oral durante a diarréia, melhorar a atenção pré e perina-
tal e a monitorização do crescimento. Mostra-se ainda que, paradoxalmente, as ações de so-
brevivência infantil concentram-se primariamente em crianças de alta renda e portanto de bai-
xo risco. Além de propiciar o planejamento e avaliação das açóes de saúde, os diagnósticos
fornecem dados basais com os quais os resultados de futuros inquéritos poderão ser comparados.

Descritores: Saúde infantil. Indicadores de saúde. Levantamentos epidemiológicos

\section{Introdução}

Na maior parte das regiões do Brasil inexistem informações confiáveis a respeito de indicadores importantes de saúde materno-infantil, tais como o estado nutricional de crianças e ges-

\footnotetext{
* Patrocinado pelo Fundo das Nações Unidas para a Infância (Unicef), pelas Secretarias de Saúde dos Estados do Ceará, Sergipe e Rio Grande do Norte e pela Secretaria Municipal de Saúde de Natal.

** Fundo das Nações Unidas para a Infância (Unicef)

*** Departamento de Medicina Social, Universidade Federal de Pelotas - Pelotas, RS - Brasil

**** Secretaria Municipal de Saúde de Fortaleza Fortaleza, CE - Brasil

$\begin{array}{ll}* * * * * & \text { Projeto Hope - Fortaleza, CE - Brasil } \\ * * * * * * & \text { Secretaria da Saúde do Estado do Ceará - Forta- }\end{array}$ leza, CE - Brasil

******* Secretaria da Saúde do Estado do Rio Grande do Norte - Natal, RN - Brasil

******** Secretaria Municipal de Saúde de Natal - Natal, RN - Brasil

********* Secretaria da Saúde do Estado de Sergipe - Aracaju, SE - Brasil

Separatas/Reprints: C.G. Victora - Departamento de Medicina Social, Universidade Federal de Pelotas - Caixa Postal
} 464 - 96100- Pelotas, RS - Brasil tantes, a duração da amamentação, a cobertura vacinal, a frequência e o manejo de doenças infecciosas como diarréia ou infecções respiratórias agudas. Tampouco existem dados válidos sobre a cobertura dos serviços de assistência pré e perinatal.

Nessas regiões, que incluem as mais pobres do país onde a morbi-mortalidade infantil atinge suas taxas mais elevadas, o conhecimento desses indicadores é essencial para um adequado planejamento das ações de saúde. Muitos dos indicadores acima nem mesmo são colhidos rotineiramente pelos serviços de saúde; quando o são, muitas vezes não alcançam a qualidade $\mathrm{e}$ a cobertura desejadas. Há necessidade, portanto, de complementá-los com diagnósticos comunitários, em que as condições de saúde e a utilização de serviços sejam avaliados em uma amostra representativa da população de uma determinada área geográfica. Se devidamente conduzidos, estes diagnósticos podem desempenhar um papel fundamental no planejamento e na avaliação dos programas e serviços de saúde ${ }^{2,9}$.

De 1987 a 1989, o Fundo das Nações Unidas para a Infância (Unicef) colaborou com or- 
gãos de saúde estaduais e municipais do Ceará, Rio Grande do Norte e Sergipe para realizar diagnósticos comunitários de saúde materno-infantil, naqueles Estados. O presente trabalho relaciona os principais achados dessas três pesquisas e aponta para possíveis medidas a serem tomadas.

\section{Metodologia}

A metodologia utilizada nos três diagnósticos estaduais foi padronizada e encontra-se descrita em detalhe em outra publicação ${ }^{2}$. Os principais aspectos metodológicos estão descritos a seguir.

O tamanho da amostra foi definido em função dos objetivos dos estudos. Para o Estado do Ceará, tinha-se como meta estimar a mortalidade infantil (dados não incluídos no presente relatório), além de indicadores de morbidade e utilização de serviços em menores de três anos. Para estimar a mortalidade pelos métodos indiretos foi necessário entrevistar um número substancial de mulheres de 15 a 49 anos ${ }^{5}$, o que exigiu que 8.000 domicílios fossem visitados. Nesses domicílios foram examinadas 4.513 crianças menores de três anos.

Nos diagnósticos do Rio Grande do Norte e Sergipe, não se tinha como objetivo avaliar mortalidade e, portanto, optou-se por uma amostra de pouco mais de 1.000 menores de cinco anos. Com esta amostra, seria possível obter estimativas dos problemas de saúde mais comuns e da cobertura de serviços, com margens aceitáveis de erro ${ }^{2}$.

Com o objetivo de obter amostras representativas das crianças menores de três (Ceará) ou cinco anos (Rio Grande do Norte e Sergipe) residentes nos Estados, utilizou-se processo de amostragem em estágios múltiplos, com três etapas. Na primeira, sorteou-se, com probabilidade proporcional à população, os municípios a serem pesquisados, na segunda os setores censitários dentro de cada município, e na terceira fase sorteou-se um ponto inicial dentro de cada setor, a partir do qual foram visitados um certo número de domicílios.

$\mathrm{Na}$ pesquisa do Ceará foram sorteados 40 municípios, 10 setores censitários em cada municicípio e 20 domicílios em cada setor, num total de 8.000 domicílios. Em Sergipe e Rio Grande do Norte, foram sorteados 20 municípios em cada Estado, oito setores censitários em cada município e 12 casas em cada setor, totalizando-se 1.920 domicílios. As capitais estaduais, em função de sua grande população, foram sorteadas várias vezes (Fortaleza 10 vezes, Aracaju 5 vezes e Natal 6 vezes). Maiores detalhes da constitui- ção das amostras encontram-se em outras publicações $2,6,7,8$.

Os trabalhos de campo foram realizados por profissionais de nível superior (enfermeiras, nutricionistas e assistentes sociais), sendo supervisionados diretamente por médicos ou enfermeiras com experiência prévia em pesquisas de campo. Os treinamentos e a coleta de dados foram supervisionados pelos coordenadores técnicos dos estudos (CGV e FCB). Os trabalhos de campo foram realizados de outubro a dezembro de 1987 (Ceará) e de novembro a dezembro de 1989 (Rio Grande do Norte e Sergipe).

O questionário aplicado à mãe ou responsável pela criança incluiu variáveis demográficas, sócio-econômicas, ambientais, nutricionais e relativas à morbidade, à utilização de serviços de saúde e aos cuidados com a criança.

As crianças foram pesadas em balanças portáteis (precisão até $100 \mathrm{~g}$ ) e seu comprimento foi medido com antropômetros AHRTAG modificados (precisão até $0,1 \mathrm{~cm}$ ), construídos localmente a partir de um modelo original. Em Sergipe e Rio Grande do Norte, crianças com mais de 2 anos de idade foram medidas em pé (estatura), com antropômetros desenvolvidos pelo Centro de Pesquisas Epidemiológicas de Pelotas. Foram consideradas como desnutridas as crianças com índices estatura (ou comprimento)/idade, peso/idade ou peso/estatura (ou comprimento) de 2 ou mais desvios-padrão abaixo do esperado, conforme a referência do National Center for Health Statistics (NCHS) ${ }^{4}$. Utilizou-se também a classificação de Gomez para peso/idade 3 .

O processamento dos dados foi realizado em micro-computador utilizando-se os programas dBase $3+$, SPSS/PC + e CASP (pacote desenvolvido pelo Center for Disease Control dos Estados Unidos da América, para análise antropométrica). Devido ao fato de que os setores rurais são menos populosos, em média, do que os setores urbanos, foi necessário utilizar um fator de ponderação para obter estimativas representativas para todo o Estado. A duração da amamentação foi calculada pelas tabelas de sobrevivência. Todas as diferenças entre os três Estados, mencionadas no texto na seção de Resultados, foram estatisticamente significativas $(p<0,05)$.

\section{Resultados}

As características das amostras nos três Estados estão descritas na Tabela 1. Em nenhum dos três diagnósticos as perdas excederam 3,5\%. Os principais motivos para perdas foram ausência dos moradores e recusas. 
Tabela 1. Características das amostras dos Estados do Ceará, Sergipe e Rio Grande do Norte.

\begin{tabular}{lccc}
\hline Estado & Ceará & Sergipe & $\begin{array}{c}\text { Rio Grande } \\
\text { do Norte }\end{array}$ \\
\hline Faixa etária & $<3$ anos & $<5$ anos & $<5$ anos \\
Número de crianças & 4.513 & 1.043 & 1.101 \\
Perdas & $0,4 \%$ & $3,3 \%$ & $1,0 \%$ \\
Ano do estudo & 1987 & 1989 & 1989 \\
\hline
\end{tabular}

A Tabela 2 mostra algumas características demográficas das amostras estudadas. As distribuições por idade e sexo estão de acordo com o esperado. Quanto à ordem de nascimento, de um quarto a um terço das crianças eram primogênitas. As ordens de nascimento mais elevadas foram mais freqüentes no Ceará do que nos demais Estados.

Tabela 2. Caracteristicas demográficas das amostras de crianças dos Estados do Cerá, Sergipe e Rio Grande do Norte.

\begin{tabular}{lccc}
\hline Característica & Ceará & Sergipe & $\begin{array}{c}\text { Rio Grande } \\
\text { do Norte }\end{array}$ \\
\hline Sexo masculino & $50,2 \%$ & $52,1 \%$ & $49,5 \%$ \\
Idade & & & \\
$\quad<1$ ano & $34,1 \%$ & $22,6 \%$ & $20,9 \%$ \\
$1-1.9$ ano & $33,8 \%$ & $21,1 \%$ & $17,3 \%$ \\
$2-2.9$ anos & $32,1 \%$ & $20,1 \%$ & $23,0 \%$ \\
$3-3.9$ anos & - & $18,6 \%$ & $18,4 \%$ \\
$4-4.9$ anos & - & $17,6 \%$ & $20,4 \%$ \\
Ordem de nascimento & & & \\
Primogênito & $25,9 \%$ & $27,5 \%$ & $31,4 \%$ \\
2 2: ou 3: & $32,7 \%$ & $39,2 \%$ & $41,8 \%$ \\
$4:$ a 6: & $22,0 \%$ & $21,8 \%$ & $17,7 \%$ \\
$\quad 7 \%$ ou mais & $19,3 \%$ & $11,5 \%$ & $9,2 \%$ \\
\hline Número de crianças & 4.513 & 1.043 & 1.101 \\
\hline
\end{tabular}

Alguns dos principais achados relativos à situação sócio-econômica e ambiental das famílias estão descritos na Tabela 3 . Estes evidenciam a situação precária em que sobrevivem grande parte das crianças nordestinas, com um perfil de baixa renda familiar, analfabetismo dos pais, habitações inadequadas e promíscuas e péssimas condições de saneamento ambiental. Em praticamente todos os indicadores estudados, o Estado do Ceará apresentou situação inferior à de Sergipe e Rio Grande do Norte, sendo as condições neste último discretamente superiores às de Sergipe.

A assistência pré e perinatal recebida pelas mães, durante a gestação das crianças incluídas na amostra, está descrita na Tabela 4. No Ceará, uma em cada três gestantes não realizou nenhuma consulta, enquanto que para os outros dois Estados esta proporção foi de uma em seis. Se considerar-mos seis consultas como o mínimo desejável, esta meta foi alcançada apenas
Tabela 3. Características sócio-econômicas e ambientais das amostras de crianças dos Estados do Ceará, Sergipe e Rio Grande do Norte.

\begin{tabular}{lccc}
\hline Característica & Ceará & Sergipe & $\begin{array}{c}\text { Rio Grande } \\
\text { do Norte }\end{array}$ \\
\hline Renda abaixo & & & \\
1 SM/mensal & $47,5 \%$ & $41,5 \%$ & $30,0 \%$ \\
Pai alfabetizado & $48,7 \%$ & $60,4 \%$ & $62,2 \%$ \\
Mãe alfabetizada & $58,4 \%$ & $62,3 \%$ & $72,8 \%$ \\
Casa de tijolo & $63,7 \%$ & $62,8 \%$ & $83,3 \%$ \\
Somente uma peça pa- & & & \\
ra dormir & $32,4 \%$ & $24,5 \%$ & $15,6 \%$ \\
Sem água encanada & $74,4 \%$ & $33,3 \%$ & $36,2 \%$ \\
Sem sanitário & $56,1 \%$ & $32,8 \%$ & $21,1 \%$ \\
Tem sabão em casa & $87,1 \%$ & $90,3 \%$ & $97,2 \%$ \\
\hline Número de crianças & 4.513 & 1.043 & 1.101 \\
\hline SM - Salário mínimo & & &
\end{tabular}

para um quarto das gestantes cearenses e por cerca de metade das sergipanas e potiguares. A cobertura com duas ou mais doses de vacina antitetânica para primíparas e pelo menos uma dose para as demais gestantes ${ }^{8}$ alcançou apenas cerca de metade das mães cearenses e sergipanas, e $70 \%$ das potiguares.

Tabela 4. Indicadores de assistência pré e perinatal nas amostras de mães e crianças dos Estados do Ceará, Sergipe e Rio Grande do Norte.

\begin{tabular}{lccc}
\hline Característica & Ceará & Sergipe & $\begin{array}{c}\text { Rio Grande } \\
\text { do Norte }\end{array}$ \\
\hline $\begin{array}{l}\text { Consultas de pré-natal } \\
\quad \text { Nenhuma }\end{array}$ & $34,8 \%$ & $15,7 \%$ & $15,7 \%$ \\
$\quad 6+$ & $24,3 \%$ & $46,4 \%$ & $52,9 \%$ \\
$\begin{array}{l}\text { Vacina antitetânica } \\
\text { (mãe) }\end{array}$ & $51,5 \%$ & $50,2 \%$ & $69,9 \%$ \\
Parto hospitalar & $64,8 \%$ & $80,4 \%$ & $89,8 \%$ \\
Parto feito por parteira & $38,0 \%$ & $40,9 \%$ & $38,6 \%$ \\
Parto por cesariana & $10,4 \%$ & $15,0 \%$ & $20,1 \%$ \\
\hline Número de crianças & 4.513 & 1.043 & 1.101 \\
\hline
\end{tabular}

* Duas doses para primiparas; uma dose para as demais gestantes.

Observou-se diferenças importantes na proporção de partos domiciliares, que variaram de cerca de $35 \%$, no Ceará, a $10 \%$, no Rio Grande do Norte. Por outro lado, a proporção de partos realizados por parteiras foi similar nos três Estados, ao redor de $40 \%$. As taxas de cesarianas variaram substancialmente, de $10 \%$, no Ceará, a 20\%, em Sergipe.

A Tabela 5 mostra que a duração do aleitamento materno nos três Estados foi bastante curta, com medianas ao redor de 3 a 4 meses, embora no Ceará houvesse uma tendência a maiores durações.

$O$ estado nutricional das crianças segundo os critérios OMS-NCHS e Gomez está descrito na Tabela 6. Os quadros de nanismo nutricional 
Tabela 5. Percentuais de crianças amamentadas ao seio conforme a idade, nas amostras de crianças dos Estados do Ceará, Sergipe e Rio Grande do Norte.

\begin{tabular}{lccc}
\hline $\begin{array}{l}\text { Percentuais de } \\
\text { crianças amamentadas }\end{array}$ & Ceará & Sergipe & $\begin{array}{c}\text { Rio Grande } \\
\text { do Norte }\end{array}$ \\
\hline 1 mês & $83,6 \%$ & $81,8 \%$ & $81,1 \%$ \\
3 meses & $62,6 \%$ & $58,0 \%$ & $58,9 \%$ \\
6 meses & $41,3 \%$ & $34,9 \%$ & $34,9 \%$ \\
12 meses & $27,1 \%$ & $23,0 \%$ & $19,5 \%$ \\
\hline Número de crianças & 4,513 & 1.043 & 1.101 \\
\hline
\end{tabular}

(déficit de altura/idade) predoninaram em relação aos déficits de peso/idade e peso/altura. A prevalência de desnutrição foi marcadamente mais elevada no Ceará do que nos demais estados, que apresentaram resultados similares. Quando as crianças com três anos ou mais foram excluídas das amostras de Sergipe e Rio Grande do Norte para permitir uma maior comparabilidade com as crianças cearenses, os déficits de peso/idade e peso/altura permaneceram praticamente inalterados, mas houve uma redução na proporção de crianças com déficit de altura/idade $-13,8 \%$ e $9,2 \%$, respectivamente, para Sergipe e Rio Grande do Norte.

A Tabela 6 mostra também a distribuição das crianças conforme o critério de Gomez, que classifica 30 a $40 \%$ das crianças como desnutridas.

Tabela 6. Prevalências de desnutrição nas amostras de crianças e mães dos Estados do Ceará, Sergipe e Rio Grande do Norte.

\begin{tabular}{lrrr}
\hline $\begin{array}{l}\text { Indicador de } \\
\text { desnutrição }\end{array}$ & Ceará & Sergipe & $\begin{array}{c}\text { Rio Grande } \\
\text { do Norte }\end{array}$ \\
\hline Crianças & & & \\
Altura/idade* & $27,6 \%$ & $16,1 \%$ & $14,2 \%$ \\
Peso/idade* & $12,8 \%$ & $7,5 \%$ & $6,2 \%$ \\
Peso/altura* & $2,4 \%$ & $3,2 \%$ & $1,3 \%$ \\
Gomez & & & \\
Normais & $60,2 \%$ & $66,5 \%$ & $69,0 \%$ \\
1.: grau & $31,8 \%$ & $28,2 \%$ & $27,0 \%$ \\
2. grau & $6,4 \%$ & $4,8 \%$ & $3,6 \%$ \\
$3^{\circ}$ grau & $1,6 \%$ & $0,5 \%$ & $0,4 \%$ \\
\hline Número de crianças & 4.513 & 1.043 & 1.101 \\
\hline * Dois desvios-padrão ou mais abaixo da mediana do pa- \\
drão NCHS.
\end{tabular}

A Tabela 7 , restrita a crianças menores de três anos, mostra a associação entre a renda familiar e as prevalências de desnutrição. Devido ao menor tamanho da amostra, os dados para Sergipe e Rio Grande do Norte apresentam maiores flutuações. Existem claras associações entre a renda familiar e as prevalências de déficits de altura/idade e peso/idade. Por outro lado, não existe tendência clara para a variável peso/altura. Em todos os grupos de renda as prevalên- cias de desnutrição tendem a ser mais elevadas no Ceará do que nos demais estados.

Tabela 7. Prevalências de desnutrição conforme renda familiar mensal nas amostras de crianças dos Estados do Ceará, Sergipe e Rio Grande do Norte. Análise restrita a crianças menores de três anos.

\begin{tabular}{|c|c|c|c|c|c|}
\hline \multirow{2}{*}{$\begin{array}{c}\text { Indicador } \\
\mathrm{e} \\
\text { Estado }\end{array}$} & \multicolumn{5}{|c|}{ Renda familiar mensal em salários mínimos } \\
\hline & $<0.5$ & $0.5-0.9$ & $1-1.9$ & $2-4.9$ & $>=5$ \\
\hline $\begin{array}{l}\text { Altura/idade* } \\
\text { CE } \\
\text { SE } \\
\text { RN }\end{array}$ & $\begin{array}{l}33,3 \% \\
26,8 \% \\
11,4 \%\end{array}$ & $\begin{array}{l}34,4 \% \\
19,6 \% \\
11,6 \%\end{array}$ & $\begin{array}{r}27,5 \% \\
7,5 \% \\
10,3 \%\end{array}$ & $\begin{array}{r}18,8 \% \\
9,9 \% \\
5,7 \%\end{array}$ & $\begin{array}{l}9,5 \% \\
1,4 \% \\
3,8 \%\end{array}$ \\
\hline $\begin{array}{l}\text { Peso/idade* } \\
\text { CE } \\
\text { SE } \\
\text { RN }\end{array}$ & $\begin{array}{r}16,2 \% \\
6,8 \% \\
9,1 \%\end{array}$ & $\begin{array}{r}16,3 \% \\
14,0 \% \\
4,6 \%\end{array}$ & $\begin{array}{r}12,3 \% \\
3,6 \% \\
9,1 \%\end{array}$ & $\begin{array}{l}8,5 \% \\
5,3 \% \\
3,5 \%\end{array}$ & $\begin{array}{l}6,0 \% \\
2,4 \% \\
2,5 \%\end{array}$ \\
\hline $\begin{array}{l}\text { Peso/Altura* } \\
\text { CE } \\
\text { SE } \\
\text { RN }\end{array}$ & $\begin{array}{l}2,5 \% \\
2,7 \% \\
0,9 \%\end{array}$ & $\begin{array}{l}2,2 \% \\
4,6 \% \\
2,9 \%\end{array}$ & $\begin{array}{l}3,0 \% \\
1,1 \% \\
2,1 \%\end{array}$ & $\begin{array}{l}2,2 \% \\
4,6 \% \\
2,2 \%\end{array}$ & $\begin{array}{l}1,5 \% \\
3,8 \% \\
1,3 \%\end{array}$ \\
\hline $\begin{array}{l}\text { Número de } \\
\text { crianças } \\
\text { CE } \\
\text { SE } \\
\text { RN }\end{array}$ & $\begin{array}{r}695 \\
103 \\
68\end{array}$ & $\begin{array}{r}1331 \\
156 \\
109\end{array}$ & $\begin{array}{r}1272 \\
133 \\
181\end{array}$ & $\begin{array}{l}619 \\
172 \\
138\end{array}$ & $\begin{array}{r}352 \\
53 \\
123\end{array}$ \\
\hline
\end{tabular}

A freqüência e manejo da diarréia estão descritos na Tabela 8. A freqüência de episódios de diarréia foi cerca de duas vezes mais alta no Ceará do que nos demais Estados. Os percentuais de utilização de terapia de reidratação oral (TRO) foram similares, atingindo apenas uma em cada quatro crianças que apresentavam diarréia. Nos diagnósticos de Sergipe e do Rio Grande do Norte, cerca de $10 \%$ das famílias dispunham de colher-medida de plástico para preparação do soro caseiro com sal e açúcar.

Tabela 8. Freqüência e manejo da diarréia nas amostras de crianças dos Estados do Ceará, Sergipe e Rio Grande do Norte.

\begin{tabular}{lcrr}
\hline Característica & Ceará & Sergipe & $\begin{array}{c}\text { Rio Grande } \\
\text { do Norte }\end{array}$ \\
\hline Presença de diarréia & & & \\
$\quad$ No dia & $12,0 \%$ & $7,3 \%$ & $6,4 \%$ \\
Nos últimos 7 dias & $26,1 \%$ & $9,3 \%$ & $8,9 \%$ \\
$\quad$ Nos últimos 15 dias & $\star$ & $15,6 \%$ & $15,4 \%$ \\
Uso de reidratante & & & \\
durante o episódio** & $22,7 \%$ & $27,8 \%$ & $21,9 \%$ \\
Possui colher-medida & $* *$ & $8,5 \%$ & $9,7 \%$ \\
\hline Número de crianças & 4.513 & 1.043 & 1.101 \\
\hline
\end{tabular}

* Informação não disponivel

* * Somente para crianças com diarréia 
Alguns indicadores de morbidade estão descritos na Tabela 9. De 7\% a $10 \%$ das crianças haviam sido hospitalizadas nos doze meses que precederam a entrevista (o valor mais elevado no Ceará pode ser devido à restrição do estudo a menores de três anos). Apesar de as crianças cearenses serem em média mais jovens, estas haviam consultado menos do que as crianças dos outros dois Estados. Isto pode ser explicado em parte pelas maiores dificuldades de acesso, uma vez que quase metade das crianças cearenses viviam a mais de cinco quilômetros de uma unidade de saúde.

Tabela 9. Alguns indicadores de morbidade e de utilização de serviços nas amostras de crianças dos Estados do Ceará, Sergipe e Rio Grande do Norte.

\begin{tabular}{lrrc}
\hline Característica & Ceará & Sergipe & $\begin{array}{c}\text { Rio Grande } \\
\text { do Norte }\end{array}$ \\
\hline $\begin{array}{l}\text { Hospitalizações últimos } \\
12 \text { meses }\end{array}$ & $9,9 \%$ & $6,2 \%$ & $6,9 \%$ \\
$\begin{array}{l}\text { Consulta nos últimos } \\
3 \text { meses }\end{array}$ & $34,9 \%$ & $49,6 \%$ & $49,0 \%$ \\
$\begin{array}{l}\text { Distância >5 km da } \\
\text { unidade de saúde }\end{array}$ & $43,1 \%$ & $18,4 \%$ & $13,5 \%$ \\
$\begin{array}{l}\text { Tosse, febre e } \\
\text { respiração difícil na }\end{array}$ & $8,6 \%$ & $6,0 \%$ & $7,1 \%$ \\
última semana & $8,5 \%$ & $11,7 \%$ & $4,6 \%$ \\
\hline História de sarampo & $8,5 \%$ & 1.043 & 1.101 \\
\hline Número de crianças & 4.513 & & \\
\hline
\end{tabular}

A Tabela 9 inclui os percentuais de crianças que haviam apresentado tosse com febre e 'respiração difícil' na semana anterior ao estudo, e aquelas que, conforme informação de suas mães, já haviam apresentado sarampo. Estas variáveis devem ser encaradas com cautela pois as informações são subjetivas. Em especial, parece que muitas das crianças com 'respiração difícil' apresentavam de fato obstrução nasal associada a infecções respiratórias altas.

Entre os dados de maior utilidade fornecidos pelos diagnósticos de saúde encontram-se os referentes à cobertura atingida pelos programas e ações preventivas (Tabela 10 ). A cobertura vacinal, aqui entendida como o percentual de crianças acima de um ano de idade que haviam recebido o número de doses recomendado para o primeiro ano, variou bastante de Estado para Estado. Das crianças cearenses, cerca de $40 \%$ haviam recebido todas as vacinas recomendadas, enquanto que para o Rio Grande do Norte este percentual foi de $70 \%$. Aceitou-se como válidas não apenas as doses registradas em carteira de vacina mas também aquelas informadas pelas mães, quando estas descreviam corretamente a técnica de imunização. Quando se restringiu a definição a doses efetivamente confirmadas pela carteira, as coberturas atingidas foram de $34,9 \%$ para o Ceará, $48,5 \%$ para Sergipe, e $60,9 \%$ para o Rio Grande do Norte.

Tabela 10. Alguns indicadores de cobertura de programas e açōes preventivas nas amostras de crianças dos Estados do Ceará, Sergipe e Rio Grande do Norte.

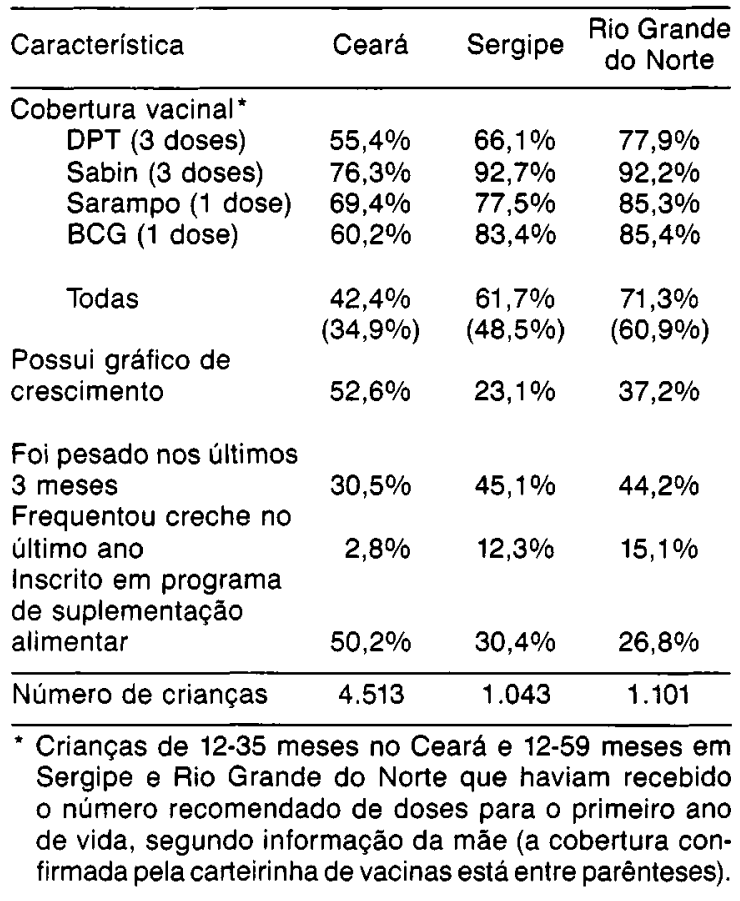

Por outro lado, mais crianças cearenses do que sergipanas ou portiguares possuiam gráfico de crescimento; isto não impediu que nos dois últimos Estados o percentual de crianças pesadas no último trimestre fosse superior $(45 \%)$ ao encontrado no Ceará $(30 \%)$. Cerca de metade das crianças cearenses estavam inscritas em algum programa de suplementação alimentar (principalmente do Instituto Nacional de Alimentação e Nutrição - $35 \%$ do total de crianças), comparadas com um quarto a um terço das crianças dos outros dois Estados.

$O$ percentual de crianças que freqüentava creches foi extremamente baixo nos três Estados, particularmente no Ceará onde atingiu apenas uma criança em cada 30 (Tabela 10).

$\mathrm{Na}$ Tabela 11, alguns dos principais indicadores de ações de sobrevivência infantil são analisados em função da renda familiar. $O$ atendimento pré-natal quantitativamente adequado, definido como um mínimo de cinco consultas durante a gestação, foi alcançado por um terço ou menos das gestantes de renda inferior a um salário mínimo e por $80 \%$ daquelas com renda superior a cinco salários. Quanto ao local do parto, metade das parturientes de baixa renda no Ceará 
e Sergipe deram à luz em um hospital, comparadas com a quase totalidade das parturientes de mais alta renda; para o Rio Grande do Norte foram também encontradas diferenças significativas mas menos marcantes.

Tabela 11. Ações de sobrevivência infantil conforme grupos de renda familiar.

\begin{tabular}{|c|c|c|c|c|c|}
\hline \multirow{2}{*}{$\begin{array}{l}\text { Indicador } \\
e \\
\text { Estado }\end{array}$} & \multicolumn{5}{|c|}{ Renda familiar mensal em salários mínimos } \\
\hline & $<0.5$ & $0.5-0.9$ & $1-1.9$ & $2-4.9$ & $>=5$ \\
\hline \multicolumn{6}{|c|}{ Pré-natal (6 ou + consultas) } \\
\hline CE & $11,1 \%$ & $11,9 \%$ & $24,7 \%$ & $42,1 \%$ & $70,3 \%$ \\
\hline SE & $22,1 \%$ & $39.1 \%$ & $45.9 \%$ & $64.1 \%$ & $64.3 \%$ \\
\hline$R N$ & $28,1 \%$ & $43,0 \%$ & $46,3 \%$ & $59,7 \%$ & $74,5 \%$ \\
\hline \multicolumn{6}{|c|}{ Parto hospitalar } \\
\hline CE & $53,1 \%$ & $50,5 \%$ & $69,7 \%$ & $82,7 \%$ & $93,7 \%$ \\
\hline SE & $50,7 \%$ & $76,3 \%$ & $82,1 \%$ & $94,5 \%$ & $98,7 \%$ \\
\hline RN & $83,9 \%$ & $85,0 \%$ & $90,2 \%$ & $93,9 \%$ & $90,9 \%$ \\
\hline \multicolumn{6}{|c|}{ Amamentação aos 6 meses } \\
\hline CE & $51,5 \%$ & $44,6 \%$ & $38,1 \%$ & $35,5 \%$ & $29,0 \%$ \\
\hline SE & $29,8 \%$ & $36,6 \%$ & $39,0 \%$ & $32,5 \%$ & $39,0 \%$ \\
\hline RN & $39,4 \%$ & $36,5 \%$ & $39,1 \%$ & $40,3 \%$ & $27,3 \%$ \\
\hline \multicolumn{6}{|c|}{ Pesadas no trimestre } \\
\hline CE & $21,2 \%$ & $26,1 \%$ & $31,0 \%$ & $37,5 \%$ & $53,1 \%$ \\
\hline SE & $33.8 \%$ & $41,5 \%$ & $50,0 \%$ & $48,8 \%$ & $51,6 \%$ \\
\hline RN & $30,5 \%$ & $36,1 \%$ & $39,9 \%$ & $51,1 \%$ & $61,1 \%$ \\
\hline \multicolumn{6}{|c|}{ Vacinas completas * } \\
\hline CE & $26,8 \%$ & $33,5 \%$ & $37,6 \%$ & $40,2 \%$ & $43,6 \%$ \\
\hline SE & $47,9 \%$ & $56,7 \%$ & $68,6 \%$ & $66,7 \%$ & $78,9 \%$ \\
\hline RN & $72,4 \%$ & $75,6 \%$ & $66,1 \%$ & $72.0 \%$ & $73,1 \%$ \\
\hline \multicolumn{6}{|c|}{ Usaram TRO** na diarréia } \\
\hline $\mathrm{CE}$ & $23,4 \%$ & $14,1 \%$ & $26,1 \%$ & $30,0 \%$ & $32,0 \%$ \\
\hline SE & $28,4 \%$ & $21,8 \%$ & $32,8 \%$ & $28,5 \%$ & $43,2 \%$ \\
\hline $\mathrm{RN}$ & $32,6 \%$ & $21,7 \%$ & $20,4 \%$ & $34,8 \%$ & $33.3 \%$ \\
\hline
\end{tabular}

* Crianças de 12-35 meses (CE) ou de $12-59$ meses (SE, RN)

* * Somente Crianças com diarréia na última semana (CE) ou nos últimos 15 dias (SE, RN).

Ao contrário dos outros indicadores incluidos na Tabela 11, a amamentação ao seio parece ser menos influenciada pelos serviços de saúde e mais por fatores sócio-culturais. Talvez por essas razões, os diferenciais por renda não foram tão claros. Para o Ceará, houve uma clara tendência para menores durações da amamentação com o aumento da renda familiar, mas para Sergipe e Rio Grande do Norte não houve diferenças marcadas.

Quanto à monitorização do crescimento e às imunizações, nos três Estados foram observadas claras tendências a maiores coberturas com o aumento da renda familiar (Tabela 11). A exceção foi a cobertura vacinal no Rio Grande do Norte, que pouco variou em função do nível sócio-econômico.

As diferenças entre os grupos de renda quanto ao uso de terapia de reidratação oral não foram marcadas, embora as maiores taxas de uso tenham sido observadas nas duas categorias de maior renda (Tabela 11). Estas estimativas podem haver sido afetadas pelo pequeno número de crianças em cada categoria, uma vez que a análise foi restrita a crianças em episódios recentes de diarréia.

\section{Discussão}

O delineamento proposto resultou na obtenção de amostras representativas das populações de crianças dos três Estados, com um reduzido percentual de crianças que deixaram de ser contactadas, o que contribui para a validade do estudo. A experiência adquirida nos diagnósticos foi extremamente valiosa e encontra-se disponivel ${ }^{2}$.

Os diagnósticos de saúde materno-infantil realizados nos três Estados do Nordeste mostraram que estas pesquisas são factíveis, relativamente baratas e fornecem dados de inestimável valia para o planejamento e avaliação de serviços de saúde. Um diagnóstico de cerca de mil crianças, como nos Estados de Sergipe e Rio Grande do Norte, pode ser realizado por uma equipe (em cada Estado) de dez entrevistadores, gastando cerca de quatro semanas em campo. Além dos gastos de pessoal, os principais ítens orçamentários incluíram transporte (dois ou três carros) e diárias para as viagens ao interior, impressão de questionários e equipamentos para antropometria (balanças e antropômetros). O custo total de um estudo de mil crianças em 15 municípios (incluindo a capital) encontra-se ao redor de US $\$ 30,000.00$, o que representa uma fração reduzida dos gastos anuais com saúde materno-infantil. Embora idealizada para aplicação em nível estadual, esta metodologia pode ser facilmente adaptada para estudos em nível de município, cidade ou bairro.

A comparação entre os três Estados, embora ilustrativa sob muitos aspectos, deve ser analisada com a devida cautela, pelas razões abaixo. Em primeiro lugar, o diagnóstico cearense foi restrito a menores de três anos, enquanto os de Sergipe e Rio Grande do Norte englobaram menores de cinco anos. Sabe-se que muitas variáveis são marcadamente afetadas pela idade, como o estado nutricional e a utilização de serviços de saúde. Nas análises acima, sempre que foi o caso, os resultados para Sergipe e Rio Grande do Norte foram apresentados apenas para menores de três anos para garantir uma maior comparabilidade. Em segundo lugar, a pesquisa cearense foi realizada dois anos antes das demais, e em particular ocorreu durante uma época seca. Isto pode haver afetado alguns indicadores, como o estado nutricional.

As informações sobre o estado nutricional 
das crianças dos três Estados são comparadas na Tabela $12 \mathrm{com}$ as de outros estudos realizados na Região Nordeste nos últimos anos. Em relação ao Estudo Nacional da Despesa Familiar (ENDEF) ${ }^{10}$, realizado em $1974-75$, todos os estudos recentes mostram uma substancial redução na prevalência de desnutrição medida pelo critério de Gomez, em particular nas formas moderadas e severas (segundo e terceiro graus). O diagnóstico do Ceará apresenta prevalência similares às encontradas na Pesquisa Nacional sobre Saúde Materno-Infantil e Planejamento Familiar (PNSMIPF) ${ }^{1}$ e à Pesquisa Nacional sobre Saúde e Nutrição (PNSN) ${ }^{5}$, particularmente se levarmos em conta que o diagnóstico do Ceará foi restrito a menores de três anos que tendem a apresentar menores prevalências do que o grupo de menores de cinco anos como um todo. No entanto, os diagnósticos de Sergipe e do Rio Grande do Norte tendem a apresentar prevalências inferiores às dos demais estudos, mesmo levando em consideração a margem de erro das estimativas. Isto sugere, diferenças metodológicas à parte, que as crianças desses dois Estados apresentam melhores condições nutricionais do que as do Nordeste como um todo.

Tabela 12. Comparação das prevalências de desnutrição (critério de Gomez) no Nordeste brasileiro em diferentes estudos.

\begin{tabular}{lllccc}
\hline \multirow{2}{*}{ Estudo } & Ano & Região & ldades & \multicolumn{2}{c}{ Prevaléncia de desnutriçăo* } \\
\cline { 5 - 6 } & & & (anos) & $\begin{array}{c}\text { Todas as } \\
\text { formas }\end{array}$ & $\begin{array}{c}\text { Formas moderadas } \\
\text { e severas }\end{array}$ \\
\hline ENDEF & 1974 & Nordeste & 1.4 & $62,4 \%$ & $21,7 \%$ \\
PNSMIPF & 1986 & Nordeste & $<5$ & $42,5 \%$ & $7,2 \%$ \\
PNSN & 1989 & Nordeste & $<5$ & $46,1 \%$ & $9,6 \%$ \\
Atual & 1987 & Ceará & $<3$ & $39,8 \%$ & $8,0 \%$ \\
& 1989 & Sergipe & $<5$ & $33,5 \%$ & $5,3 \%$ \\
& 1989 & RG Norte & $<5$ & $31,0 \%$ & $4,0 \%$ \\
\hline
\end{tabular}

"Classificação de Gomez

Os indicadores obtidos pelos diagnósticos contribuíram para o planejamento e avaliação das ações sanitárias. Entre outras medidas, apontam para a necessidade de incentivar 0 aleitamento materno, aumentar a cobertura vacinal, incrementar o uso da TRO durante a diarréia, melhorar a assistência pré e perinatal e a monitorização do crescimento.

A existência de um extenso banco de dados coletados através do diagnóstico fornece também informaçðes basais com as quais estudos subseqüentes podem ser comparados. Por exemplo, foi recentemente concluído em 1990, no Estado do Ceará, um segundo estudo, utilizando exatamente a mesma amostragem e metodologia do diagnóstico de 1987, o qual mostrou importantes progressos nos indicadores de saúde materno- infantil*. Os resultados dos diagnósticos comunitários, se devidamente devolvidos às populações pelos meios de comunicações de massa, podem servir ainda para instrumentalizar a sociedade civil em sua luta por melhores condições de saúde.

Em resumo, os dados fornecidos pelos três diagnósticos mostram que uma fração substancial das mães e crianças nordestinas vive sob condições de carência extrema, as quais em última análise são responsáveis pelo padrão de morbi-mortalidade observado. Demonstram também que os recentes esforços na promoção de estratégias de sobrevivência infantil, como as imunizações, a terapia de reidratação oral, a promoção do aleitamento e a monitorização do crescimento, embora promissores, ainda apresentam coberturas insatisfatórias. Um resultado particularmente preocupante foi a concentração das ações de sobrevivência infantil entre as crianças de maior renda familiar, e portanto de menor risco de morbi-mortalidade. São necessários esforços marcados no sentido de impedir que as açðes de saúde, ao invés de promover a equiidade, contribuam para uma ainda maior polarização nas desigualddes sociais.

VICTORA, C.G. et al [Child health in the States of Ceará, Rio Grande do Norte and Sergipe, Brazil: description of a methodology for the diagnosis of communities]. Rev. Saúde públ., S. Paulo, 25: 218-25, 1991. No reliable data are available from most Brazilian states for a number of child health indicators, such as nutritional status, breast feeding, vaccine coverage, incidence and management of infectious diseases, and coverage of antenatal and perinatal services. How ever a methodology has been developed for state-wide, community-based health surveys for the obtaining of such information, which was recently applied in studies of representative samples of children from the states of Ceará, Sergipe and Rio Grande do Norte in Northeastern Brazil. The present report describes the key aspects of this methodology and some of the main findings. These results point out to the need for promoting breast feeding, increasing vaccine coverage, improving diarrhoea management with oral rehydration therapy and investing in antenatal and perinatal care, as well as in growth monitoring. They also show that child survival activities are paradoxically concentrated on higher-income, lower risk children. Besides contributing to the planning and evaluation of health programs, community-based child health surveys provide baseline data against which future progress may be ascertained.

Keywords: Child health. Health status indicators. Health surveys.

\section{Referências Bibliográficas}

1. ARRUDA, J.M.; RUTHNBERG, N.; MORRIS, L.; FERRAZ, E.A. Pesquisa nacional sobre saúde materno-infantil e planejamento familiar (PNSMIPF), Brasil 1986. Rio de Janeiro, BEMFAM/IRD, 1987.

\footnotetext{
* Secretaria da Saúde do Estado do Ceará - Dados inéditos
} 
2. BARROS, F.C.\& VICTORA, C.G. Epidemiologia da saúde infantil: um manual para diagnósticos comunitários. São Paulo, Hucitec, Unicef, 1991.

3. GOMEZ, F.; GALVAN, R.R.; FRENK, S.; CRAVIOTO MUNOS J.; CHAVEZ, R.; VASQUEZ, J. Mortality in second and third degree malnutrition. $J$. trop. Pediat., 2:77-83, 1956.

4. HAMILL, R.V.V.; DRIZD, T.A.; JOHNSON, C.L.; REED, R.B.; ROCHE, A.F. NCHS growth curves for children, birth-18 years. Vital Hith Statist. Ser., 11, (165) 1977.

5. INSTITUTO DE ALIMENTAÇÃO E NUTRIÇÃO. Pesquisa nacional sobre saúde e nutrição: resultados preliminares. Brasília. INAN/IBGE/IPEA, 1990.

6. INSTITUTO DE PLANEJAMENTO DO CEARÁ/FUNDO DAS NAÇŌES UNIDAS PARA A INFÂNCIA (UNICEF). A saúde das crianças cearenses: $u m$ estudo de 8000 famílias. Fortaleza, IPLANCE, 1990.

7. SECRETARIA DE ESTADO DA SAÚDE DE SERGIPE. Fundação Estadual de Planejamento, Pesquisa e Estatistica/FUNDO DAS NAÇŌES UNIDAS PARA A INFÂNCIA (UNICEF). Crianças $e$ adolescentes em Sergipe: saúde, educação e trabalho. Aracajú, 1990.
8. SECRETARIA ESTADUAL DE SAÚDE DO RIO GRANDE DO NORTE/ SECRETARIA MUNICIPAL DE SAÚDE DE NATAL/FUNDO DAS NAÇÕES UNIDAS PARA A INFÂNCIA (UNICEF). Crianças $e$ adolescentes no Rio Grande do Norte: saúde, educação e trabalho, Natal, 1990.

9. VAUGHAN, J.P.; MORROW, R. Epidemiology for district health planning. Geneva, World Health Organization, 1988.

10. VIACAVA, F.; FIGUEIREDO, C.M.P.; OLIVEIRA, W.A. A desnutrição no Brasil: uma análise do Estudo Nacional da Despesa Familiar - (IBGE 74/75) para o Nordeste, Estado de São Paulo e Rio de Janeiro. Petrópolis, Vozes, 1983.

11. WORLD HEALTH ORGANIZATION. Expanded Programme on Immunizations. Prevention of neonatal tetanus through immunization. Geneva, 1986.

Recebido para publicação $23 / 11 / 1990$ Aprovado para publicação em 13/4/199I 